\title{
The CUrious Variables Experiment (CURVE). Variable properties of the dwarf nova SS Ursae Minoris ${ }^{\star}$
}

\author{
A. Olech ${ }^{1}$, K. Mularczyk ${ }^{2}$, P. Kędzierski ${ }^{2}$, K. Złoczewski ${ }^{2}$, M. Wiśniewski ${ }^{1}$, and K. Szaruga ${ }^{2}$ \\ 1 Nicolaus Copernicus Astronomical Center, ul. Bartycka 18, 00-716 Warszawa, Poland \\ e-mail: olech,mwisniew@camk . edu.pl \\ 2 Warsaw University Observatory, Al. Ujazdowskie 4, 00-478 Warszawa, Poland \\ e-mail: [kmularcz;pkedzier;kzlocz]@astrouw.edu.pl
}

Received 7 November 2005 / Accepted 18 January 2006

\section{ABSTRACT}

\begin{abstract}
We report on extensive photometry of the dwarf nova SS Ursae Minoris throughout nine months of 2004. In total, we recorded two superoutbursts and 11 normal outbursts of the star. SS UMi has been known to show frequent superoutbursts with a mean interval of 84.7 days. Our data suggest that the interval between successive superoutbursts lengthened to 197 days, indicating that SS UMi entered a period of untypical behavior manifested by a growth in the quiescent magnitude of the star and a series of frequent, low-amplitude, normal outbursts observed from July to September 2004.

The mean superhump period derived for the April 2004 superoutburst of SS UMi is $P_{\mathrm{sh}}=0.070149$ (16) days $(101.015 \pm 0.023 \mathrm{~min})$. Combining this value with an earlier orbital period determination, we were able to derive the period excess, which is equal to $3.5 \pm$ $1.6 \%$, and estimate the mass ratio of the binary system as equal to $q=0.16 \pm 0.07$.

During the entire superoutburst, the period decreased at a rate of $\dot{P} / P_{\mathrm{sh}}=-6.3(1.4) \times 10^{-5}$. However, detailed analysis of the timings of superhump maxima seem to suggest a more complex period change, with a decrease in the period during the first and last stages of the superoutburst but an increase in the middle interval.
\end{abstract}

Key words. stars: binaries: close - stars: variables: general - stars: dwarf novae

\section{Introduction}

SS Ursae Minoris (PG 1551 +719) was identified as a cataclysmic variable candidate in the Palomar Green Survey (Green et al. 1982) and, almost at the same time was discovered, as an X-ray source E1551 +718 (Mason et al. 1982).

The quiescent spectra of the star taken by Mason et al. (1982) showed broad and strong hydrogen and helium emission lines typical of dwarf novae. The HeII 4686 emission line was weak and the object had no detectable polarization (Green et al. 1982), which suggested that the star has no strong magnetic field and thus is a typical nonmagnetic dwarf nova. The broad emission lines have double peaked structure, indicating high inclination and thus the possible presence of an orbital wave in the light curve from quiescence.

The photometric behavior of SS UMi was investigated by Andronov (1986), who observed the object photographically on 77 plates. In total, eight possible eruptions were detected. The photographic photometry of SS UMi was analyzed further by Richter (1989). He found that the height of the maxima varies between 13.2 and $14.3 \mathrm{mag}$ and that the star shows two types of eruptions: long and short. The fact that faint maxima were sometimes longer than bright ones suggested that SS UMi belongs to U Gem class rather than SU UMa type variables.

The first CCD photometry of SS UMi was reported by Udalski (1990), who found clear light modulations in the quiescence but no eclipses. The two possible periods were 6.8 and $9.5 \mathrm{~h}$ suggesting that one of them might be an orbital period of

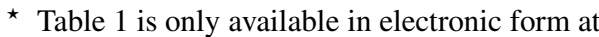
http: //WwW .edpsciences.org the binary system and thus SS UMi is in fact a U Gem type star. Photometric measurements of SS UMi were also obtained by Chen et al. (1991) who observed two outbursts of the star. Detection of the superhumps, i.e. 0.3 mag amplitude characteristic tooth-shape light modulations during eruptions from March and September 1989 clearly showed that SS UMi belongs to the SU UMa class of dwarf novae. Chen et al. (1991) estimated the period of the superhumps to be around $101 \mathrm{~min}$.

Time-resolved specroscopy of SS UMi and six other SU UMa stars was obtained by Thorstensen et al. (1996). The variations in the positions of $\mathrm{H} \alpha$ emission lines allowed determination of the orbital period of the binary, which is equal to $P_{\text {orb }}=0.06778$ days $(97.6 \pm 1.5 \mathrm{~min})$.

Another superoutburst of SS UMi was observed by Kato et al. (1998). They observed the star on four nights of April 1998 eruption. During two nights the star showed clear superhumps with a mean period of $P_{\mathrm{sh}}=0.0699 \pm 0.0003$ days $(100.7 \pm 0.4 \mathrm{~min})$.

Kato et al. (2000) analyzed 375 visual and CCD observations of SS UMi taken from February 18, 1999 to June 17, 2000 and reported by VSNET observers. Inspection of the global light curve allowed them to find that the intervals between successive superoutbursts are in the range of 82-86 days with a mean value of 84.7 days. They also noticed that there are five normal outbursts bewteen two successive superoutbursts, meaning that the mean cycle length is around 11 days.

\section{ER UMa stars}

The first members of the ER UMa class objects were discovered in the mid 1990s (ER UMa, RZ LMi, V1159 Ori, and DI UMa). 
These are systems characterized by an extremely short supercycle (20-60 days), a short interval between normal outbursts (3-4 days), and small amplitude ( $\sim 3 \mathrm{mag}$ ) of superoutbursts (Kato \& Kunjaya 1995; Robertson et al. 1995; Patterson et al. 1995). Subsequently, one more object with similar characteristics was discovered (IX Dra - Ishioka et al. 2001; Olech et al. 2004a).

These objects seemed to be very unusual compared to normal SU UMa stars. Ten years ago, the ordinary SU UMa star with the shortest supercycle of 134 days was YZ Cnc (Patterson 1979; Shafter \& Hessman 1988). Thus supercycles of ER UMa stars were about 3-4 times shorter. However, when describing the results of the CBA observational campaign for V1159 Ori, Patterson et al. (1995) claimed that there is no reason to introduce a new class of variable stars. Simply, the observable traits of ER UMa-type stars seem to be consistent with garden-variety SU UMa stars. They follow the Kukarkin-Parengo relation connecting the amplitude of the outburst with recurrence time between normal outbursts and the Bailey relation connecting decay times from the normal eruptions and orbital period of the binary. They simply appear to be normal SU UMa stars with greater activity and greater luminosity due to their higher-mass transfer rates (Osaki 1996).

However, careful inspection of the diagram with recurrence intervals for supermaxima vs. normal maxima showed a significant gap between normal SU UMa stars and ER UMa-type variables (see Fig. 18 of Paterson et al. 1995).

As we can see, SS UMi with a supercycle of 85 days lies in the transition area between extremely active dwarf novae and normal SU UMa stars. Investigating the behavior and characteristics of this star might help us to better understand this class of objects. This was the reason for including SS UMi into the primary targets observed within the CURVE (Olech et al. 2003a,b). In this work we report the results of a nine month observational campaign performed in 2004.

\section{Observations and data reduction}

Observations of SS UMi reported in the present paper were obtained during 88 nights between April 13, 2004 and December 8, 2004 at the Ostrowik station of the Warsaw University Observatory. The data was collected using the $60-\mathrm{cm}$ Cassegrain telescope equipped with a Tektronics TK512CB back-illuminated CCD camera. The scale of the camera was $0.76^{\prime \prime} /$ pixel providing a $6.5^{\prime} \times 6.5^{\prime}$ field of view. The full description of the telescope and camera was given by Udalski and Pych (1992). We monitored the star in "white light". We did not use any filters in order to shorten the exposures to minimize guiding errors, due to the temporary lack of an autoguiding system, after a recent telescope renovation. The exposure times were from 100 to $150 \mathrm{~s}$ during the bright state and from 180 to $300 \mathrm{~s}$ at minimum light.

A full journal of our CCD observations of SS UMi is given in Table 1. In total, we monitored the star during $105.374 \mathrm{~h}$ and obtained 2021 exposures. All the data reductions were performed using a standard procedure based on the IRAF $^{1}$ package and the profile photometry was derived using the DAOphotII package (Stetson 1987).

${ }^{1}$ IRAF is distributed by the National Optical Astronomy Observatory, which is operated by the Association of Universities for Research in Astronomy, Inc., under a cooperative agreement with the National Science Foundation.

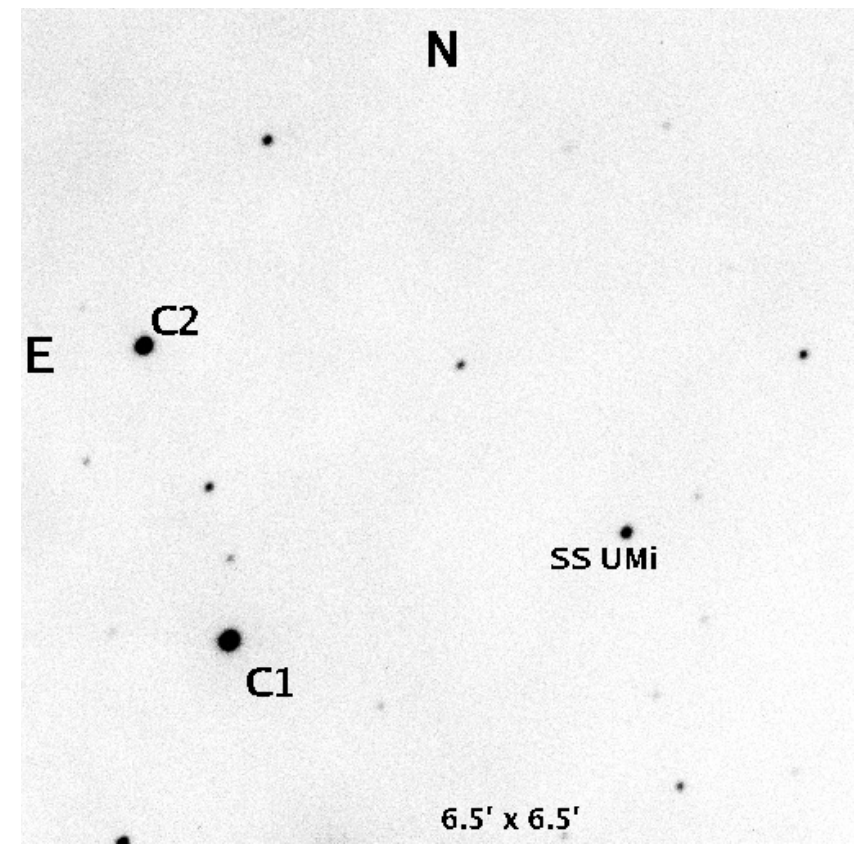

Fig. 1. Finding chart for SS UMi covering a region of $6.5 \times 6.5 \mathrm{arcmin}$. The positions of the comparison stars are shown. North is up, East is left.

Relative, unfiltered magnitudes of SS UMi were determined as the difference between the magnitude of the variable and the intensity averaged magnitude of the two comparison stars: $\mathrm{C} 1\left(\mathrm{RA}=15^{\mathrm{h}} 51^{\mathrm{m}} 59.5^{\mathrm{s}}\right.$, Dec $=+71^{\circ} 44^{\prime} 25.6^{\prime \prime}, V=12.553, B-$ $V=0.883)$ and C2 $\left(\mathrm{RA}=15^{\mathrm{h}} 52^{\mathrm{m}} 07.3^{\mathrm{s}}, \mathrm{Dec}=+71^{\circ} 46^{\prime} 34.6^{\prime \prime}\right.$, $V=13.226, B-V=1.052)$. The comparison stars and the variable are marked in the chart displayed in Fig. 1. The basic properties of comparison stars are taken from the catalog of Henden \& Honeycutt (1997).

The typical accuracy of our measurements varied between 0.006 and 0.024 mag in the bright state and between 0.009 and 0.2 mag in the minimum light. The median value of the photometry errors was 0.009 and 0.045 mag, respectively.

\section{Global light curve}

Almost all our observations were made in "white light" in order to obtain precise photometry of the star in quiescence. However, on the night of April 22/23, 2004 we also made $V, R$, and $I$ photometry of the variable and comparison stars. Using the magnitude data from Henden \& Honeycutt (1995), we were able to transform our instrumental magnitudes to the standard $V$ system.

Such a transformed global light curve of SS UMi spanning eight months of observations is shown in Fig. 2, which shows both our own CCD observations and those in the visual made by American Association of Variable Stars Observers (AAVSO), including their CCD magnitude estimates and also the upper magnitude limits in case of non detection of the variable. It is clear that the brightness of SS UMi varies from around 17.2 mag at quiescence to around $13.7 \mathrm{mag}$ at the maximum of superoutburst. During the brightest normal outbursts the star can be found at a magnitude of around 14.4.

The vertical arrows point to the maximum brightness of the two superoutbursts clearly visible in the light curve. They are separated by 197 days, which is much longer than the mean supercycle length of 84.7 days determined by Kato et al. (2000). 


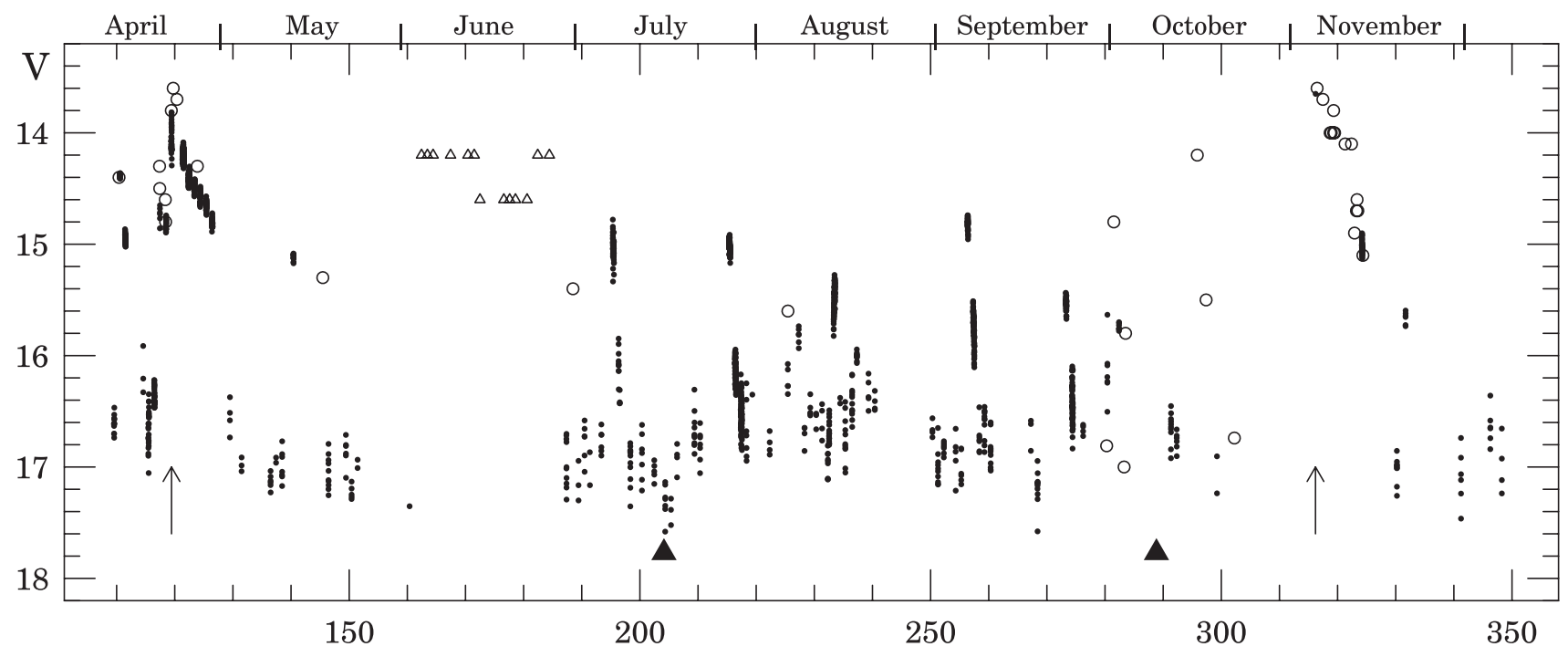

HJD - 2453000

Fig. 2. The general photometric behavior of SS UMi during the whole 2004 campaign. Visual estimates collected by AAVSO observers are shown as open circles and the CCD observations described in this paper as black dots. The open triangles correspond to the "fainter than" estimates from AAVSO. Big filled triangles point to the expected positions of superoutbursts assuming 84.7 days supercycle length. Two arrows show the moments of maximum magnitude of the recorded superoutbursts.

As we can see, the lack of our CCD observations during June nights, HJD from 2453161 to 2453187 , may suggest that another superoutburst occurred in that time. This is, however, unlikely for two reasons. First, it would suggest that the supercycle of SS UMi shortened to about 50 days, and second, the observations of AAVSO clearly indicate that during June nights the star was fainter than 14.2-14.6 mag and thus was able to show only ordinary outbursts but no superoutburst. The big filled triangles in Fig. 2 denote the expected moments of maxium brightness of the missing superoutbursts assuming a 84.7-day supercycle of SS UMi.

\section{April 2004 superoutburst}

The April 2004 superoutburst started on Apr. 20/21 with a normal outburst, the so-called precursor. After an initial rise in brightness, the magnitude of the star decreased slightly and then the tidal instability triggered the superoutburst manifested by the appearance of the superhumps and an increase in the brightness. Starting with the night of Apr. 23/24, SS UMi entered the plateau phase. During this period clear superhumps were present and the brightness of the star was decreasing at a rate of $0.12 \mathrm{mag} / \mathrm{day}$. Around May 1st, the magnitude started to decrease much faster, indicating termination of the superoutburst. On May 4/5 the star was again at its quiescent magnitude.

\subsection{Superhumps}

The light curves from individual nights of the April 2004 superoutburst of SS UMi are shown in Fig. 3. Clear superhumps were present in the light curve of the star from Apr. 22/23 to Apr. 30/01. During the first night, before reaching maximum brightness, the amplitude of the superhumps was only $0.13 \mathrm{mag}$. A night later, the superhumps were fully developed with their characteristic tooth-shape and an amplitude of $0.33 \mathrm{mag}$. On Apr. 24/25 the shape of the superhumps did not change, but the amplitude decreased to $0.15-0.20 \mathrm{mag}$ depending on the hump. The next night the amplitude stayed at the same level, but clear secondary humps became visible. In the interval Apr. 26/27Apr. 28/29, the amplitude of the light variations was around $0.15 \mathrm{mag}$ and secondary humps became more pronounced. On Apr. 30/01 the superhumps had a smaller amplitude than $0.1 \mathrm{mag}$ and after that night the plateau phase ended.

From each light curve of SS UMi in superoutburst, we removed the first or second order polynomial and analyzed them using ANOva statistics with two harmonic Fourier series (Schwarzenberg-Czerny 1996). The resulting periodogram is shown in Fig. 4. The most prominent peak is found at a frequency of $f=14.238 \pm 0.020 \mathrm{c} / \mathrm{d}$, which corresponds to the period of $P_{\mathrm{sh}}=0.07023(10)$ days $(101.13 \pm 0.14 \mathrm{~min})$. The harmonic peak at $28.5 \mathrm{c} / \mathrm{d}$ is real and leads to the presence of secondary humps in the light curve.

From each light curve of the superoutburst we removed variablility corresponding to the superhump period. Such a prewhitened light curve was again analyzed by ANOva statistics. No significant peak was recorded in the computed power spectrum.

\subsection{The $O-C$ analysis}

To check the stability of the superhump period and to determine its value, we constructed an $\mathrm{O}-\mathrm{C}$ diagram. We decided to use the timings of primary maxima, because they were almost always high and clearly detectable in the light curve of the variable. In the end, we were able to determine 17 moments of maxima, which are listed in Table 2 together with their errors, cycle numbers $E$, and $\mathrm{O}-\mathrm{C}$ values.

The least-square linear fit to the data from Table 2 gives the following ephemeris for the maxima:

$\mathrm{HJD}_{\max }=2453118.5294(12)+0.070147(17) \times E$.

The $\mathrm{O}-\mathrm{C}$ values computed according to the ephemeris (1) are listed in Table 2 and also shown in Fig. 5. It is clear that the superhump period shows a slightly decreasing trend, confirmed by the second-order polynomial fit to the moments of maxima, 


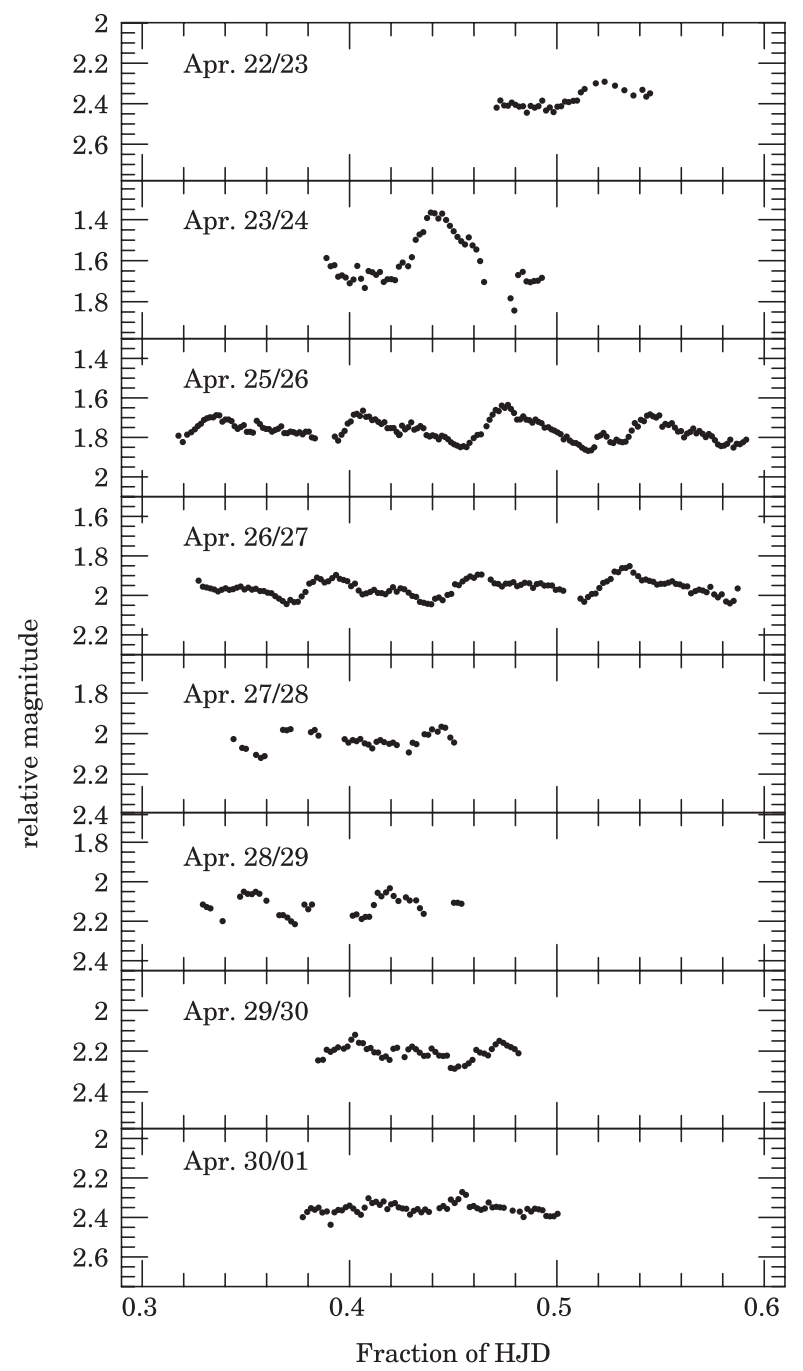

Fig. 3. The light curves of SS UMi during its April 2004 superoutburst.

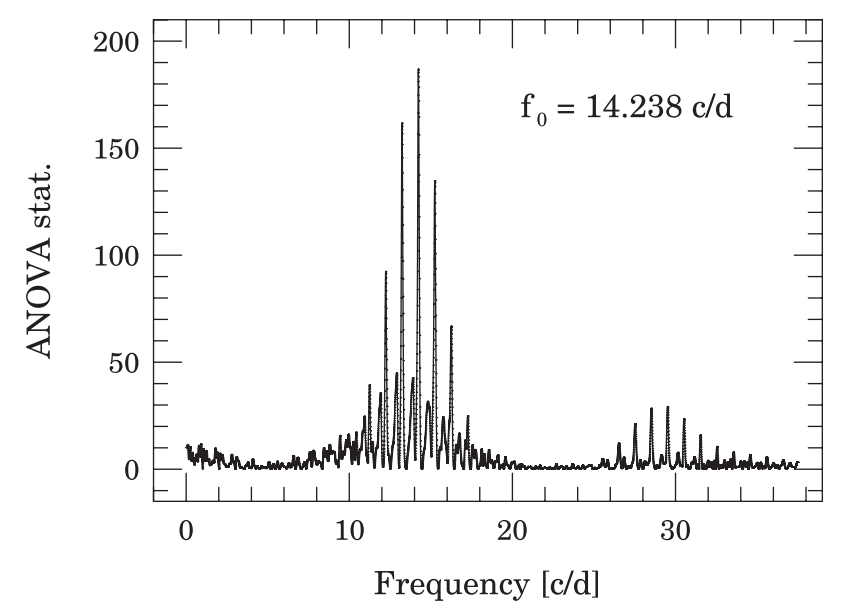

Fig. 4. The ANOVA power spectrum of the light curve of SS UMi from its 2004 April superoutburst.

which gives the following ephemeris (shown as a solid line in Fig. 5):

$$
\begin{array}{r}
\mathrm{HJD}_{\max }=2453118.5240(17)+0.070413(60) \times E \\
-2.22(0.48) \times 10^{-6} \times E^{2} .
\end{array}
$$

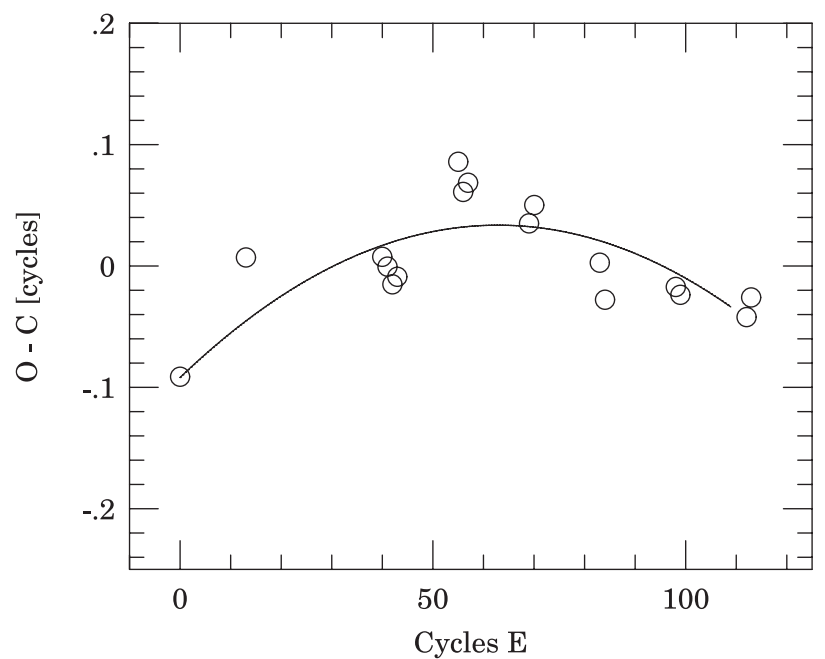

Fig. 5. The O-C diagram for the superhump maxima of SS UMi during its April 2004 superoutburst. The solid line corresponds to the fit resulting from the ephemeris (2).

Table 2. Timings of maxima in the light curve of SS UMi during its 2004 April superoutburst.

\begin{tabular}{cccc}
\hline \hline Cycle $E$ & HDJ -2453000 & Error & O-C \\
\hline 0 & 118.5230 & 0.0020 & -0.0912 \\
13 & 119.4418 & 0.0025 & +0.0070 \\
40 & 121.3358 & 0.0030 & +0.0074 \\
41 & 121.4054 & 0.0035 & -0.0004 \\
42 & 121.4745 & 0.0022 & -0.0153 \\
43 & 121.5451 & 0.0025 & -0.0088 \\
55 & 122.3935 & 0.0020 & +0.0857 \\
56 & 122.4619 & 0.0025 & +0.0608 \\
57 & 122.5326 & 0.0020 & +0.0687 \\
69 & 123.3720 & 0.0035 & +0.0350 \\
70 & 123.4432 & 0.0030 & +0.0500 \\
83 & 124.3518 & 0.0025 & +0.0028 \\
84 & 124.4198 & 0.0023 & -0.0278 \\
98 & 125.4026 & 0.0015 & -0.0172 \\
99 & 125.4723 & 0.0018 & -0.0236 \\
112 & 126.3829 & 0.0030 & -0.0422 \\
113 & 126.4542 & 0.0020 & -0.0258 \\
\hline
\end{tabular}

Finally, we conclude that the period of the superhumps was not stable during the April 2004 superoutburst of SS UMi; and in the interval covered by our observations it is roughly described by a decreasing trend with a rate of $\dot{P} / P_{\mathrm{sh}}=-6.3(1.4) \times 10^{-5}$. The combination of both our superhump period determinations returned its mean value to $P_{\mathrm{sh}}=0.070149$ (16) days $(101.015 \pm$ $0.023 \mathrm{~min})$.

\section{Discussion}

\subsection{Basic parameters of the binary system}

Knowing the exact value of the superhump period and orbital period of the binary system determined by Thorstensen et al. (1996), we can compute the period excess defined as $\Delta \epsilon=\left(P_{\mathrm{sh}}-\right.$ $\left.P_{\text {orb }}\right) / P_{\text {orb }}$. In the case of SS UMi it is equal to $3.5 \pm 1.6 \%$. The relatively large error comes mainly from the $1.5 \mathrm{~min}$ error in the Thorsensen et al. (1996) determination of the orbital period.

The obtained value is typical for the SU UMa stars with orbital periods of around $98 \mathrm{~min}$, indicating that SS UMi follows the 
Stolz \& Schoembs (1984) relation between the period excess and orbital period of the binary.

Superhumps occur at a period slightly longer than the orbital period of the binary system. They are most probably the result of accretion disc precession caused by the gravitational perturbations from the secondary. These perturbations are most effective when the disc particles moving on eccentric orbits enter the 3:1 resonance. Then the superhump period is simply the beat period between orbital and precession rate periods (Osaki 1996).

Assuming the known disk size at 3:1 resonance, it is easy to correlate the mass ratio of the system $q=M_{\text {secondary }} / M_{\mathrm{WD}}$ with the period excess $\epsilon$ :

$\epsilon \approx \frac{0.23 q}{1+0.27 q}$

This relation allows estimation of the mass ratio $q$ in the dwarf nova system based only on observational determination of the orbital and superhump periods. In the case of SS UMi, this mass ratio is equal to $0.16 \pm 0.07$.

\subsection{Superhump period change}

Until the mid 1990's, all members of the SU UMa group seemed to show only negative superhump period derivatives (Warner 1995). This was interpreted as a result of disk shrinkage during the superoutburst, thus lengthening its precession rate (Lubow 1992). This picture became more complicated when the first stars with $\dot{P}>0$ were discovered. Positive period derivatives were observed only in stars with short superhump periods close to the minimum orbital period for hydrogen rich secondary (e.g. SW UMa - Semeniuk et al. 1997; WX Cet - Kato et al. 2001a; HV Vir - Kato et al. 2001b) or for stars below this boundary (e.g. V485 Cen - Olech 1997; 1RXS J232953.9+062814 - Uemura et al. 2002).

Two years ago, Olech et al. (2003a) investigated the O-C diagrams for KS UMa, ER UMa, V1159 Ori, CY UMa, V1028 Cyg, RZ Sge, and SX LMi and claimed that most (probably almost all) SU UMa stars show decreasing superhump periods at the beginning and the end of superoutburst but increasing period in the middle phase. This hypothesis was quickly confirmed by observations of the June 2004 superoutburst of TT Boo, which showed the following period derivatives $(-52.3 \pm$ $1.3) \times 10^{-5},(12.3 \pm 4.8) \times 10^{-5}$ and $(-6.2 \pm 0.9) \times 10^{-5}$, from the beginning to end of the superoutburst, respectively (Olech et al. 2004b).

Careful inspection of Fig. 5 suggests that in the case of SS UMi, we encounter the same situation as in TT Boo. The $\mathrm{O}-\mathrm{C}$ residuals may be fitted with simple parabola, as in Fig. 5, but it is clear that this fit is only a rough estimation of global behavior. In fact, it is safer to say that in the case of the April 2004 superoutburst of SS UMi, the timings of superhump maxima seem to suggest more complex period change, with a decrease in the period during the first and last stages of the superoutburst, but an increase in the middle interval. The fitting of parabolas to the to the cycle intervals $0-43,13-57$, and $40-113$ gives the period derivatives of $(-54 \pm 21) \times 10^{-5},(+27 \pm 12) \times 10^{-5}$, and $(-7.5 \pm 3.7) \times 10^{-5}$, respectively. As we can see, these values are quite similar to the preiod derivatives determined for corresponding phases of the superoutburst of TT Boo (Olech et al. 2004b).

Very recently, Uemura et al. (2005) have suggested that superhump period change might be connected with a presence of the precursor in the light curve of the superoutburst. In the

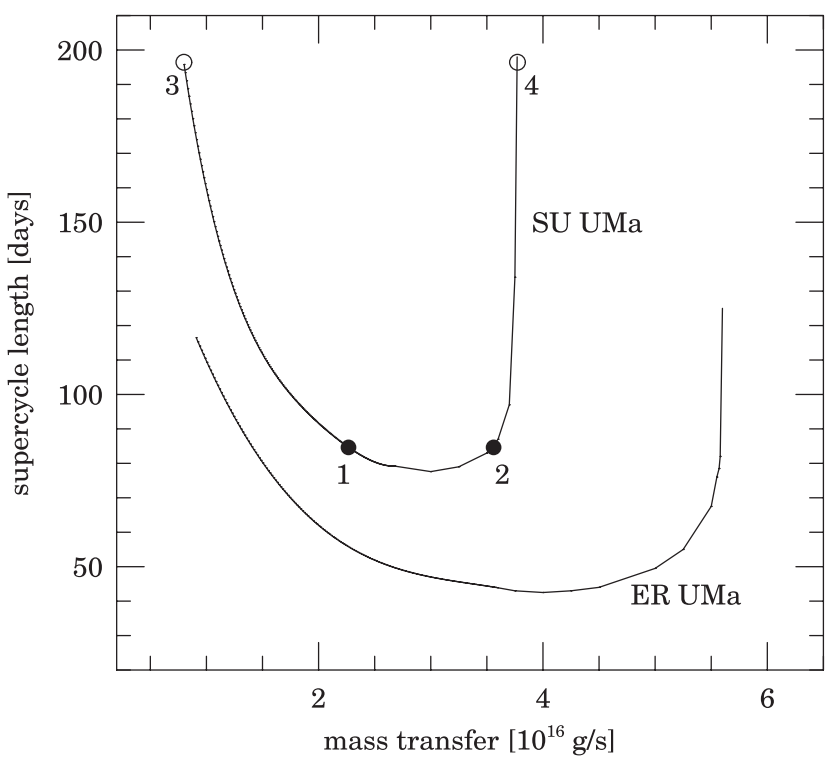

Fig. 6. The dependences between supercycle length and mass transfer in normal SU UMa stars and ER UMa variables.

case of a superoutburst without the precursor, superhump period derivatives tend to be larger than those in precursor-type eruptions. The precursor-type April 2004 superoutburst of SS UMi, characterized by large period changes similar to these observed in TT Boo, seems not to fit the scenario proposed by Uemura et al. (2005).

\subsection{Supercycle length and mass transfer}

In normal SU UMa stars, the dependence of the supercycle length $t_{\mathrm{S}}$ on the mass transfer rate $\dot{M}$ is an U-shaped curve with a broad minimum at 80-85 days (Osaki 1995a,b). In fact, the supercycle length $t_{\mathrm{S}}$ consists of two parts: $t_{\mathrm{d}}$, which is the duration of the superoutburst, and $t_{\text {wait }}$, which corresponds to the time between the end of the superoutburst and begining of the succesive superoutburst. In ordinary SU UMa stars, $t_{\text {wait }}$ is typically around one hundred days and $t_{\mathrm{d}}$ is between 10 and 15 days.

With increasing mass transfer $\left(\dot{M}>3 \times 10^{16} \mathrm{~g} \mathrm{~s}^{-1}\right.$ but still below the critical value above which the star becomes a permanent superhumper), the supercycle starts to lengthen again. However, this time it is not due to the long $t_{\text {wait }}$ but to the long duration of the superoutburst $t_{\mathrm{d}}$. This is caused by the quasi-steady state of the accretion disk, because mass-transfer rate $\dot{M}$ approaches very near to the mass accretion rate from the disk to the primary.

The standard thermal-tidal instability model is unable to produce stars with supercycles shorter than 80 days. Thus for explaining the behavior of ER UMa stars, with supercycles between 20 and 60 days, Osaki (1995a,b) assumed that the tidal torques are weaker in such systems. This results in a shorter duration of the superoutburst and larger disk radius at its termination as an effect of less angular momentum removed during such a short superoutburst. The angular momentum reservoir of the disk could then be refilled in a shorter time and another superoutburst might start more quickly.

Figure 6 shows the U-shaped dependence between $t_{\mathrm{S}}$ and $\dot{M}$ for normal SU UMa dwarf novae and ER UMa stars taken from Osaki (1995a,b). The filled circles denoted with numbers " 1 " and "2" correspond to the two possible positions of SS UMi for a supercycle of 84.7 days. The open circles marked by 


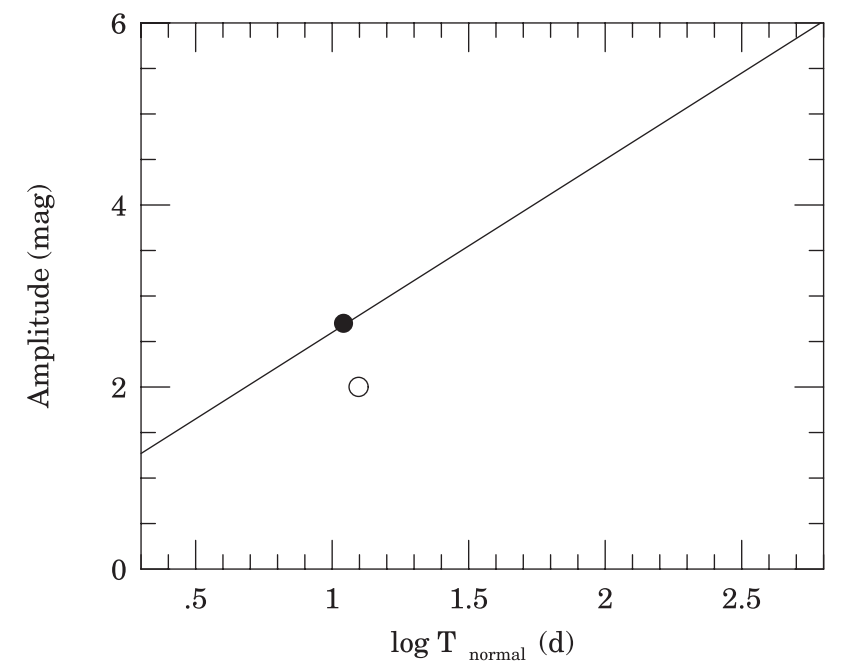

Fig. 7. The Kukarkin-Parenago relation between amplitude of the outburst and cycle length for dwarf novae. The filled and open circles denote the positions of SS UMi for 1999-2000 and 2004, respectively.

numbers " 3 " and "4" denote the possible positions of SS UMi after lengthening of the supercycle to 197 days.

The possible scenarios for SS UMi correspond to the transitions $1 \rightarrow 4,2 \rightarrow 4,1 \rightarrow 3$ and $2 \rightarrow 3$. The first two are consistent with a mass-transfer rate increasing very close to the critical limit above which a star becomes a permanent superhump object. In this case, the lengthening of the supercycle length should be caused by the long duration of the superoutbursts. This contradicts our observations, which show that both April and November superoutbursts lasted about 15 days.

On the other hand, the transitions $1 \rightarrow 3$ and $1 \rightarrow 2$ are consistent with $t_{\mathrm{d}}$ and $t_{\text {wait }}$ times indicated by the global light curve of SS UMi shown in Fig. 2. But in this case, we should assume the decrease in the mass transfer rate by a factor of $2-4$. It is not justified by the behavior of the star between superoutbursts, when we observed an increase in the quiescent magnitude by about $0.2-0.3$ mag and increased frequency of normal eruptions, which was most probably the reaction of the system to enhanced mass flow to the accretion disk. The question why the star expelled the matter by a series of frequent and low-amplitude normal outbursts observed from July to September 2004, and not by one or two superoutbursts, remains open.

\subsection{Another possibility for SS UMi?}

Another question arising in the case of SS UMi concerns its typical state. It might be that SS UMi is a normal SU UMa star with a supercycle length of 197 days and behavior observed in 1999-2000, with a supercycle of 85 days, was atypical.

According to Kato et al. (2000), in 1999-2000 SS UMi had a supercycle of 84.7 days, a cycle of 11.0 days, and a mean amplitude of normal outbursts of $2.7 \mathrm{mag}$. In 2004, it switched to a supercycle of 197 days. Due to the gaps in the observational coverage, we can not estimate the normal cycle length precisely. But between the superoutbursts from April and November, we recorded at least 11 normal outbursts with a mean amplitude of 2.0 mag. Taking the length of the gaps into account, we estimate that the number of normal eruptions could reach the level of 15 , indicating a cycle length of around 12-13 days.

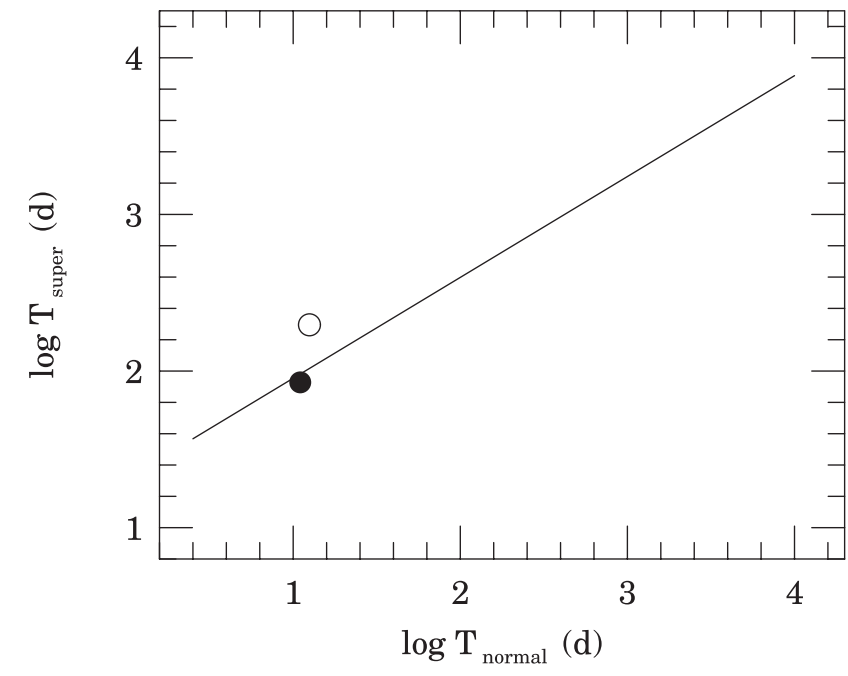

Fig. 8. The relation between amplitude supercycle and cycle lengths for dwarf novae. The filled and open circles denote the positions of SS UMi for 1999-2000 and 2004, respectively.

The amplitudes of the dwarf novae follow the famous Kukarkin-Parenago relation (Kukarkin \& Parenago 1934; Warner 1987) in the form:

$A_{\mathrm{n}}(\mathrm{mag})=0.70+1.90 \log T_{\mathrm{n}}(\mathrm{d})$

where $A_{\mathrm{n}}$ is an amplitude of normal outburst and $T_{\mathrm{n}}$ is a mean interval between two successive eruptions. Figure 7 shows this relation as a solid line. It is clear that only the 1999-2000 behavior fits the Kukarkin-Parenago relation well.

Another empirical relation followed by dwarf novae connects their cycle and supercycle lenghts (Patterson et al. 1995; Warner 1995; Olech et al. 2004a):

$\log T_{\mathrm{s}}=1.31+0.644 \log T_{\mathrm{n}}$.

Figure 8 shows this relation and again the state from 1999-2000 seems to be more typical for dwarf novae than behavior from 2004.

\section{Summary}

The nine-month observational campaign of SS UMi performed in 2004 allowed us to draw the following conclusions:

1. The supercycle of SS UMi lengthened from 84.7 days in 1999-2000 to 197 days in 2004, indicating that SS UMi entered a period of atypical behavior manifested by a growth in the quiescent magnitude of the star and series of frequent, low amplitude normal outbursts observed from July to September 2004.

2. Detailed photometry during the April 2004 superoutburst of SS UMi allowed us to determine its superhump period to be equal to $P_{\mathrm{sh}}=0.070149$ (16) days.

3 . The combination of the superhump and orbital period determinations allowed us to derive the period excess, which is equal to $\epsilon=3.5 \pm 1.6 \%$, and estimate the mass ratio of the binary system as equal to $q=0.16 \pm 0.07$.

4. The superhump period was not constant during the April 2004 superoutburst of SS UMi, and its behavior is consistent with the scenario of decreasing superhump period in the first and last stages of superoutburst but increasing in the middle. 
5. All observational properties of SS UMi can be explained using the standard thermal-tidal instability model without assuming weaker tidal torques as is necessary in describing the behavior of ER UMa stars.

Acknowledgements. We would like to thank the referee Prof. Yoji Osaki for valuable remarks and pointing out the mistake in our interpretation. We are also grateful to Prof. Józef Smak and Dr. Grzegorz Stachowski for reading and commenting on the manuscript. We gratefully acknowledge the generous allocation of time at the Warsaw Observatory 0.6-m telescope. This work was partially supported by KBN grant number 1 P03D 00627 to A. Olech and used the on-line service of the AAVSO.

\section{References}

Andronov, I. L. 1986, Astron. Tsirk., 1432, 7

Chen, J.-S., Liu, X.-W., \& Wei, M.-Z. 1991, A\&A, 242, 397

Green, R. F., Ferguson, D. H., \& Liebert, J. 1982, PASP, 94, 560

Henden, A. A., \& Honeycutt, R. K. 1997, PASP, 109, 441

Ishioka, R., Kato, T., Uemura, M., et al. 2001, PASJ, 53, L51

Kato, T., \& Kunjaya, C. 1995, PASJ, 47, 163

Kato, T., Lipkin, Y., Retter, A., \& Leibowitz, E. 1998, IBVS No. 4602

Kato, T., Hanson, G., Poyner, G., et al. 2000, IBVS No. 4932

Kato, T., Masumoto, K., Nogami, D., Morikawa, K., \& Kiyota, S. 2001a, PASJ, 53,893

Kato, T., Sekine, T., \& Hirata, R. 2001b, PASJ, 53, 1191

Kukarkin, B. V., \& Parenago, P. P. 1934, Var. Star Bull., 4, 44

Lubow, S. H. 1992, ApJ, 401, 317
Mason, K. O., Reichert, G. A., Bowyer, S., \& Thorstensen, J. R. 1982, PASP, 94, 521

Olech, A. 1997, Acta Astron., 47, 281

Olech, A., Schwarzenberg-Czerny, A., Kȩdzierski, P., et al. 2003a, Acta Astron., 53, 175

Olech, A., Kẹdzierski, P., Złoczewski, K., Mularczyk, K., \& Wiśniewski, M. 2003b, A\&A, 411, 483

Olech, A., Złoczewski, K., Mularczyk, K., et al. 2004a, Acta Astron., 54, 57

Olech, A., Cook, L. M., Złoczewski, K., et al. 2004b, Acta Astron., 54, 233

Osaki, Y. 1995a, PASJ, 47, L11

Osaki, Y. 1995b, PASJ, 47, L25

Osaki, Y. 1996, PASP, 108, 39

Patterson, J. 1979, AJ, 84, 804

Patterson, J., Jablonski, F., Koen, C., O’Donoghue, D., \& Skillman, D. R. 1995, PASP, 107, 1183

Richter, G. A. 1989, Astron. Nachr., 310, 143

Robertson, J. W., Honeycutt, R. K., \& Turner, G. W. 1995, PASP, 107, 443

Schwarzenberg-Czerny, A. 1996, ApJ, 460, L107

Semeniuk, I., Olech, A., Kwast, T., \& Należyty, M. 1997, Acta Astron., 47, 201

Shafter, A. W., \& Hessman, F. V. 1988, AJ, 95, 178

Stetson, P. B. 1987, PASP, 99, 191

Stolz, B., \& Schoembs, R. 1984, A\&A, 132, 187

Thorstensen, J. R., Patterson, J. O., Shambrook, A., \& Thomas, G. 1996, PASP, 108,73

Udalski, A. 1990, IBVS No. 3425

Udalski, A., \& Pych, W. 1992, Acta Astron., 42, 285

Uemura, M., Kato, T., Ishioka, I., et al. 2002, PASJ, 54, 599

Uemura, M., Mennickent, R. E., Ishioka, R., et al. 2005, A\&A, 432, 261

Warner, B. 1987, MNRAS, 227, 23

Warner, B. 1995, Cataclysmic Variable Stars (Cambridge University Press) 
A. Olech et al.: CURVE. Variable properties of the dwarf nova SS UMi, Online Material $p 1$

\section{Online Material}


A. Olech et al.: CURVE. Variable properties of the dwarf nova SS UMi, Online Material $p 2$

Table 1. Journal of the CCD observations of SS UMi.

\begin{tabular}{|c|c|c|c|c|c|c|c|c|c|}
\hline $\begin{array}{l}\text { Date of } \\
2004\end{array}$ & $\begin{array}{c}\text { Start } \\
2453000 .+\end{array}$ & $\begin{array}{c}\text { End } \\
2453000 .+\end{array}$ & $\begin{array}{c}\text { Length } \\
{[\mathrm{hr}]}\end{array}$ & $\begin{array}{l}\text { No. of } \\
\text { frames }\end{array}$ & $\begin{array}{l}\text { Date of } \\
2004\end{array}$ & $\begin{array}{c}\text { Start } \\
2453000 .+\end{array}$ & $\begin{array}{c}\text { End } \\
2453000 .+\end{array}$ & $\begin{array}{c}\text { Length } \\
{[\mathrm{hr}]}\end{array}$ & $\begin{array}{l}\text { No. of } \\
\text { frames }\end{array}$ \\
\hline Apr. 13/14 & 109.54367 & 109.60431 & 1.455 & 14 & Jul. 31/01 & 218.31734 & 218.34645 & 0.699 & 7 \\
\hline Apr. $14 / 15$ & 110.52498 & 110.60747 & 1.980 & 43 & Aug. 01/02 & 219.32961 & 219.35228 & 0.544 & 12 \\
\hline Apr. $15 / 16$ & 111.44819 & 111.55250 & 2.503 & 54 & Aug. 04/05 & 222.32545 & 222.33127 & 0.140 & 4 \\
\hline Apr. $18 / 19$ & 114.56803 & 114.57752 & 0.228 & 3 & Aug. $07 / 08$ & 225.43890 & 225.45064 & 0.282 & 5 \\
\hline Apr. $19 / 20$ & 115.44451 & 115.55515 & 2.655 & 27 & Aug. 09/10 & 227.31842 & 227.32588 & 0.179 & 6 \\
\hline Apr. $20 / 21$ & 116.46420 & 116.53175 & 1.621 & 36 & Aug. 10/11 & 228.31429 & 228.32109 & 0.163 & 4 \\
\hline Apr. $21 / 22$ & 117.43697 & 117.45411 & 0.411 & 6 & Aug. $11 / 12$ & 229.30221 & 229.31089 & 0.208 & 5 \\
\hline Apr. $22 / 23$ & 118.47092 & 118.54493 & 1.776 & 32 & Aug. $12 / 13$ & 230.31946 & 230.32380 & 0.104 & 3 \\
\hline Apr. 23/24 & 119.38887 & 119.49275 & 2.493 & 51 & Aug. 13/14 & 231.32677 & 231.33327 & 0.156 & 4 \\
\hline Apr. $25 / 26$ & 121.31750 & 121.59130 & 6.571 & 178 & Aug. $14 / 15$ & 232.31815 & 232.57177 & 1.356 & 27 \\
\hline Apr. 26/27 & 122.32719 & 122.58720 & 6.240 & 137 & Aug. $15 / 16$ & 233.32523 & 233.58060 & 6.129 & 101 \\
\hline Apr. $27 / 28$ & 123.34403 & 123.45042 & 2.553 & 37 & Aug. 16/17 & 234.44161 & 234.44564 & 0.097 & 2 \\
\hline Apr. 28/29 & 124.32926 & 124.45396 & 2.993 & 39 & Aug. $17 / 18$ & 235.31037 & 235.33822 & 0.668 & 11 \\
\hline Apr. $29 / 30$ & 125.38484 & 125.48146 & 2.319 & 51 & Aug. 18/19 & 236.48867 & 236.51599 & 0.656 & 13 \\
\hline Apr. 30/01 & 126.37744 & 126.50035 & 2.950 & 64 & Aug. $19 / 20$ & 237.30917 & 237.32350 & 0.344 & 10 \\
\hline May $03 / 04$ & 129.48034 & 129.49041 & 0.242 & 4 & Aug. 21/22 & 239.31643 & 239.32572 & 0.223 & 5 \\
\hline May $05 / 06$ & 131.48946 & 131.49493 & 0.131 & 3 & Aug. $22 / 23$ & 240.40505 & 240.41100 & 0.143 & 4 \\
\hline May $10 / 11$ & 136.47427 & 136.48934 & 0.362 & 6 & Sep. 01/02 & 250.31094 & 250.31941 & 0.203 & 4 \\
\hline May $11 / 12$ & 137.42191 & 137.42374 & 0.044 & 2 & Sep. 02/03 & 251.26665 & 251.28535 & 0.449 & 10 \\
\hline May $12 / 13$ & 138.44843 & 138.47103 & 0.542 & 7 & Sep. 03/04 & 252.27148 & 252.28902 & 0.421 & 7 \\
\hline May $14 / 15$ & 140.41082 & 140.43849 & 0.664 & 16 & Sep. $05 / 06$ & 254.30278 & 254.32018 & 0.418 & 5 \\
\hline May $20 / 21$ & 146.43194 & 146.45168 & 0.474 & 10 & Sep. 06/07 & 255.26395 & 255.27509 & 0.267 & 6 \\
\hline May $23 / 24$ & 149.43084 & 149.44656 & 0.377 & 6 & Sep. $07 / 08$ & 256.30955 & 256.45321 & 3.448 & 40 \\
\hline May $24 / 25$ & 150.41066 & 150.41786 & 0.173 & 5 & Sep. 08/09 & 257.29443 & 257.53091 & 5.676 & 91 \\
\hline May $25 / 26$ & 151.44124 & 151.44550 & 0.102 & 2 & Sep. 09/10 & 258.37591 & 258.39313 & 0.413 & 7 \\
\hline Jun. 03/04 & 160.38724 & 160.39526 & 0.192 & 5 & Sep. $10 / 11$ & 259.25465 & 259.27890 & 0.582 & 11 \\
\hline Jun. 30/01 & 187.35596 & 187.38250 & 0.637 & 10 & Sep. 11/12 & 260.31552 & 260.33897 & 0.563 & 11 \\
\hline Jul. 02/03 & 189.44094 & 189.44500 & 0.097 & 3 & Sep. $18 / 19$ & 267.24640 & 267.24861 & 0.053 & 3 \\
\hline Jul. 03/04 & 190.47962 & 190.49031 & 0.257 & 6 & Sep. $19 / 20$ & 268.38386 & 268.41340 & 0.709 & 11 \\
\hline Jul. 04/05 & 191.38762 & 191.40979 & 0.532 & 2 & Sep. $24 / 25$ & 273.25110 & 273.37382 & 2.945 & 50 \\
\hline Jul. 06/07 & 193.36622 & 193.37928 & 0.313 & 6 & Sep. $25 / 26$ & 274.31794 & 274.47224 & 3.703 & 64 \\
\hline Jul. 08/09 & 195.33973 & 195.53558 & 4.700 & 98 & Sep. $27 / 28$ & 276.22740 & 276.23427 & 0.165 & 4 \\
\hline Jul. 09/10 & 196.33697 & 196.53760 & 0.568 & 14 & Oct. $01 / 02$ & 280.39856 & 280.41151 & 0.311 & 6 \\
\hline Jul. 11/12 & 198.34706 & 198.36742 & 0.489 & 12 & Oct. 03/04 & 282.39762 & 282.41202 & 0.346 & 7 \\
\hline Jul. 13/14 & 200.35101 & 200.36211 & 0.266 & 7 & Oct. $12 / 13$ & 291.33376 & 291.35430 & 0.493 & 10 \\
\hline Jul. 15/16 & 202.46961 & 202.47820 & 0.206 & 5 & Oct. $13 / 14$ & 292.32704 & 292.33975 & 0.305 & 6 \\
\hline Jul. 17/18 & 204.34832 & 204.36139 & 0.314 & 7 & Oct. $20 / 21$ & 299.25359 & 299.25545 & 0.044 & 2 \\
\hline Jul. 18/19 & 205.34856 & 205.35433 & 0.138 & 3 & Nov. $06 / 07$ & 316.22524 & 316.22569 & 0.011 & 1 \\
\hline Jul. 19/20 & 206.39249 & 206.40227 & 0.235 & 4 & Nov. $14 / 15$ & 324.17099 & 324.26325 & 2.214 & 50 \\
\hline Jul. 22/23 & 209.36131 & 209.39549 & 0.820 & 10 & Nov. $20 / 21$ & 330.19057 & 330.21009 & 0.468 & 9 \\
\hline Jul. 23/24 & 210.34668 & 210.35964 & 0.311 & 7 & Nov. $21 / 22$ & 331.68761 & 331.69816 & 0.253 & 6 \\
\hline Jul. 28/29 & 215.32681 & 215.54904 & 5.333 & 148 & Dec. $01 / 02$ & 341.21800 & 341.23103 & 0.313 & 6 \\
\hline Jul. 29/30 & 216.33435 & 216.56104 & 5.441 & 89 & Dec. $06 / 07$ & 346.26354 & 346.27634 & 0.307 & 6 \\
\hline Jul. 30/31 & 217.33269 & 217.55530 & 5.343 & 92 & Dec. 08/09 & 348.24499 & 348.25133 & 0.152 & 4 \\
\hline
\end{tabular}

